\title{
A RARE CAUSE OF A TREATABLE PANCREATIC HEAD MASS WITH
}

GENERALIZED LYMPHADENOPATHY: IgG4-RELATED DISEASE

M.H. Gharib, E.H. satti, A.W. Al-Allaf Hamad Medical Corporation, Medicine/Rheumatology,

IgG4 related disorder is a new evolving immune-mediated disease, with wide range of clinical features involving multiple systems, which could mimic malignancies and lymphoma. The diagnosis depends on clinical and characteristic histopathology findings.

We report 58-years old Malaysian gentleman with background history of hypertension, CKD, who presented with slowly progressing painless cervical lymphadenopathy, parotids enlargement, orbital swelling with no fever, night sweats or weight loss. He has been treated with various antibiotics with no response.

Ultrasound neck revealed salivary and parotid glands enlargement with lymphadenopathy. FNA from lymph node was inconclusive. Follow up ultrasounds showed progression of his lymphadenopathy.

Then he was seen in Rheumatology department for possible Sjogren's syndrome, which was ruled out with negative immunology. Infections such as EBV, CMV, HIV and tuberculosis were all excluded. Lymphoma was suspected, and then ruled out by hematologist.

A year after extensive investigations, the patient was admitted with painless obstructive jaundice with negative viral and autoimmune hepatitis screen. MRCP revealed: Pancreatic head mass obstructing the bile ducts, enlarged peri-pancreatic lymph nodes, multiple parenchymal lesions of both kidneys and focal Aortitis. MRCP reported possible lymphoma, pancreatic malignancy or IgG4-related disease. Pancreatic malignancy ruled out by EUS- FNA biopsy. Lymphoma excluded by excisional LN biopsy with negative Flow cytometry. However, it showed increased nodal IgG4 plasma cells (>30/hpf), consistent with IgG4-related disease. Subsequently, blood test showed elevated IgG-4 supporting the histological diagnosis of IgG4 disease.

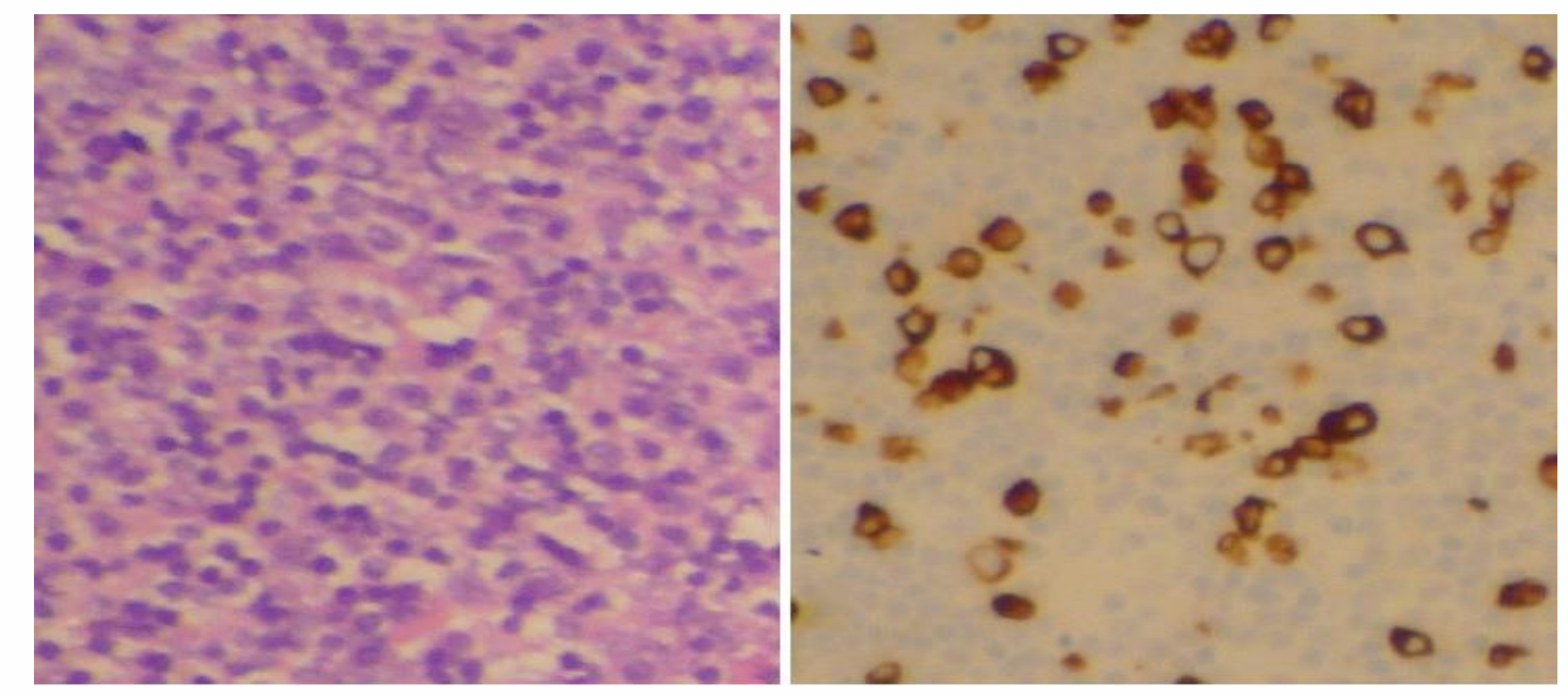

Hematoxylin and eosin staining (a) and immunohistochemical staining (b) of lymph node tissue showing numerous IgG4-positive plasma cells.

The patient responded very well to IV pulses followed by oral steroid with complete resolution of his jaundice and lymphadenopathy and his IgG4 dropped towards normal.

High index of suspicion is essential for early diagnosis and management of such disease, which if left untreated could lead to irreversible fibrosis and organ damage. It is essential to consider this diagnosis in patients with possible lymphoma and other malignancies. 